\title{
Light meson mass dependence of the positive parity heavy-strange mesons
}

\author{
Martin Cleven ${ }^{1}$, Feng-Kun Guo ${ }^{1}$, Christoph Hanhart ${ }^{1,2}$, and Ulf-G. Meißner ${ }^{1,2,3}$ \\ ${ }^{1}$ Institut für Kernphysik and Jülich Center for Hadron Physics, Forschungszentrum Jülich, D-52425 Jülich, Germany \\ ${ }^{2}$ Institute for Advanced Simulation, Forschungszentrum Jülich, D-52425 Jülich, Germany \\ ${ }^{3}$ Helmholtz-Institut für Strahlen- und Kernphysik and Bethe Center for Theoretical Physics, Universität Bonn, \\ D-53115 Bonn, Germany
}

Address(es) of author(s) should be given

the date of receipt and acceptance should be inserted later

\begin{abstract}
We calculate the masses of the resonances $D_{s 0}^{*}(2317)$ and $D_{s 1}(2460)$ as well as their bottom partners as bound states of a kaon and a $D^{(*)}$ - and $B^{(*)}$-meson, respectively, in unitarized chiral perturbation theory at next-to-leading order. After fixing the parameters in the $D_{s 0}^{*}(2317)$ channel, the calculated mass for the $D_{s 1}(2460)$ is found in excellent agreement with experiment. The masses for the analogous states with a bottom quark are predicted to be $M_{B_{s 0}^{*}}=(5696 \pm$ 40) $\mathrm{MeV}$ and $M_{B_{s 1}}=(5742 \pm 40) \mathrm{MeV}$ in reasonable agreement with previous analyses. In particular, we predict $M_{B_{s 1}}-M_{B_{s 0}^{*}}=46 \pm 1 \mathrm{MeV}$. We also explore the dependence of the states on the pion and kaon masses. We argue that the kaon mass dependence of a kaonic bound state should be almost linear with slope about unity. Such a dependence is specific to the assumed molecular nature of the states. We suggest to extract the kaon mass dependence of these states from lattice QCD calculations.
\end{abstract}

PACS. 12.39.Fe Chiral Lagrangians - 13.75.Lb Meson-meson interactions - 14.40.Lb Charmed mesons

\section{Introduction}

The discovery of two narrow resonances with open charm in 2003 opened a new chapter in hadronic spectroscopy. The $D_{s 0}^{*}(2317)$ was discovered by the BaBar collaboration [1] and shortly after the $D_{s 1}(2460)$ by the CLEO collaboration [2] Both were confirmed later.

These resonances were not in agreement with the predictions of constituent quark models, for instance the Godfrey-Isgur model [3, that on the other hand were very successful for all other open charm states discovered so far. The masses of the $D_{s 0}^{*}(2317)$ and of the $D_{s 1}(2460)$ are approximately $160 \mathrm{MeV}$ and $70 \mathrm{MeV}$ respectively, below the predictions in Ref. [3]. In addition,

$$
M_{D_{s 1}(2460)}-M_{D_{s 0}^{*}(2317)} \simeq M_{D^{*}}-M_{D} .
$$

This equality is difficult to explain in a conventional quark model. It can be explained in a parity doubling model 415. This result is preserved at one-loop

\footnotetext{
${ }^{1}$ BaBar already observed a signal of the $D_{s 1}(2460)$ in [1].
}

level [6. However, it is not clear if the parity doubling is a consequence of quantum chromodynamics (QCD). The equality of mass splittings appears naturally, if both states are assumed to be molecular states of a kaon and a $D$ or a $D^{*}$ [7], since spin-dependent interactions for a heavy quark are suppressed by two powers of $\Lambda_{\mathrm{QCD}} / m_{Q}$ (see discussion in Section 2), with $\Lambda_{\mathrm{QCD}}$ the soft QCD scale and $m_{Q}$ the mass of the heavy quark. Soon after the discovery, based on phenomenological calculations, Barnes and Close 8 and van Beveren and Rupp 9] pointed out the possible molecular nature of these resonances: they described them as bound states of $D^{(*)} K$ mesons. For reviews of possible explanations of these states, see Refs. [1011. The purely phenomenological calculations were shortly after improved by Refs. [12131415], where variants of unitarized chiral perturbation theory were used, and in addition open bottom molecules were also studied. In Refs. 1617] both resonances emerged as molecules from Weinberg-Tomozawa type interactions using flavor$\mathrm{SU}(4)$ constraints.

Clearly it is important to find observables that allow one to identify the nature of the mentioned states 
model-independently either from data or from a comparison to lattice QCD results. Up to now a few such quantities were pointed out, namely the value of the hadronic width of the resonances [18,19|20] — which in a molecular assignment turns out to be significantly larger than that for a quark state 21 — and the value of the $K D$ scattering length in the resonance channel 22] - which gets large and negative, if the states are molecules 2324. In this work we add an additional quantity, namely the quark mass and especially the kaon mass dependence of the resonance masses. We demonstrate that this dependence is qualitatively different for quark states and for hadronic molecules and thus, a study of this dependence using lattice QCD is of high importance.

Our study is based on Refs. 20 22, but extended in that now $D$ - and $D^{*}$-mesons are both included on equal footing. This allows us to also investigate the implications of heavy quark spin symmetry.

The paper is organized as follows. In Section 2 we give the Lagrangian for and display the calculation of Goldstone boson scattering off $D$ - and $D^{*}$ mesons as well as the unitarization scheme used to generate the $D_{s 0}^{*}(2317)$ and the $D_{s 1}(2460)$ as hadronic molecules. In Section 3 the coupled channel problem in the strangeness $S=1$ isoscalar channel is solved and the $D_{s 0}^{*}(2317)$ and the $D_{s 1}(2460)$ are generated as $D K$ and $D^{*} K$ molecules. We discuss the extrapolation of their masses to unphysical values for the pion and kaon mass, respectively. A brief summary and outlook is given in Section 4 . The appendix contains some details on the derivation of the pertinent scattering amplitudes.

\section{Scattering amplitudes}

The leading order (LO) Lagrangian in Heavy Meson Chiral Perturbation Theory is given by the kinetic energy of the heavy fields, the coupling of the heavy fields to pions and the mass splitting of the heavy mesons 25]26]27.

$$
\begin{aligned}
\mathscr{L}_{\mathrm{LO}}= & -i \operatorname{Tr}\left[\bar{H}_{a} v_{\mu} D_{b a}^{\mu} H_{b}\right]+g_{\pi} \operatorname{Tr}\left[\bar{H}_{a} H_{b} \gamma_{\nu} \gamma_{5}\right] u_{b a}^{\nu} \\
& +\frac{\lambda}{m_{Q}} \operatorname{Tr}\left[\bar{H}_{a} \sigma_{\mu \nu} H_{a} \sigma^{\mu \nu}\right]
\end{aligned}
$$

with the heavy fields defined as 28

$$
H=\frac{1+\psi}{2}\left[V+i P \gamma_{5}\right], \quad \bar{H}=\gamma^{0} H^{\dagger} \gamma^{0},
$$

where Latin indices denote flavor and the trace is taken over the Gamma matrices. Here, the heavy fields are $P=\left(D^{0}, D^{+}, D_{s}^{+}\right)$and $V_{\mu}=\left(D_{\mu}^{* 0}, D_{\mu}^{*+}, D_{s, \mu}^{*+}\right)$, and $v_{\mu}=(1, \mathbf{0})+\mathscr{O}(\mathbf{p} / M)$ is the heavy mesons velocity. The covariant derivative is

$$
D_{\mu}=\partial_{\mu}+\Gamma_{\mu}, \quad \Gamma_{\mu}=\frac{1}{2}\left(u^{\dagger} \partial_{\mu} u+u \partial_{\mu} u^{\dagger}\right)
$$

where

$$
U=\exp \left(\frac{\sqrt{2} i \phi}{F}\right), \quad u^{2}=U
$$

with $F$ the pion decay constant in the chiral limit. Up to the order we are working, $F$ can be replaced by the physical pion decay constant $F_{\pi}=92.4 \mathrm{MeV}$. As we will see, it is basically $F$ that provides the strength of the interaction.

The Goldstone boson fields are collected in the matrix $\phi$ with

$$
\phi=\left(\begin{array}{ccc}
\frac{1}{\sqrt{2}} \pi^{0}+\frac{1}{\sqrt{6}} \eta & \pi^{+} & K^{+} \\
\pi^{-} & -\frac{1}{\sqrt{2}} \pi^{0}+\frac{1}{\sqrt{6}} \eta & K^{0} \\
K^{-} & \bar{K}^{0} & -\frac{2}{\sqrt{6}} \eta
\end{array}\right) .
$$

The axial-vector current is defined as

$$
u_{\mu}=i u^{\dagger}\left(\partial_{\mu} U\right) u^{\dagger}
$$

The low-energy constant (LEC) $g_{\pi}$ can be fixed from the strong decay of the $D^{*+}$. Taking the values from the Particle Data Group (PDG) 29], we find $g_{\pi}=$ $0.30 \pm 0.03$.

The spin-symmetry breaking term gives rise to a mass difference

$$
\Delta=M_{V}-M_{P}=-8 \frac{\lambda}{m_{Q}},
$$

with $m_{Q}$ the pertinent heavy quark mass. In this work we use physical values for the meson masses and thus the mentioned term is considered automatically. Note, since $M_{D_{s} *}-M_{D_{s}} \neq M_{D *}-M_{D}$ (c.f. Eqs. (10)), in this way we also include an effect of simultaneous spin symmetry and SU(3) violation, which is formally of next-to-next-to leading order $\left(\mathrm{N}^{2} \mathrm{LO}\right)$. We come back to the quantitative role of this subleading effect below.

The Lagrangian at next-to-leading order (NLO) is

$$
\begin{aligned}
\mathscr{L}_{\mathrm{NLO}}= & +\operatorname{Tr}\left[\overline { H } _ { a } \left(-h_{0}\left(\chi_{+}\right)_{b b}+h_{2}\left(u_{\mu} u^{\mu}\right)_{b b}\right.\right. \\
& \left.\left.+h_{4}\left(-i v_{\mu}\right)\left(u^{\mu} u^{\nu}\right)_{b b}\left(i v_{\nu}\right)\right) H_{a}\right] \\
& +\operatorname{Tr}\left[\bar{H}_{a}\left(h_{1}\left(\tilde{\chi}_{+}\right)_{b a}+h_{3}\left(u_{\mu} u^{\mu}\right)_{b a}\right) H_{b}\right] \\
& +h_{5} \operatorname{Tr}\left[\bar{H}_{a}\left(-i v_{\mu}\right)\left\{u^{\mu}, u^{\nu}\right\}_{b a}\left(i v_{\nu}\right) H_{b}\right],
\end{aligned}
$$

where we have introduced explicit chiral symmetry breaking terms due to non-vanishing light quark masses using

$$
\begin{aligned}
& \chi_{ \pm}=u^{\dagger} \chi u^{\dagger} \pm u \chi^{\dagger} u \\
& \tilde{\chi}_{ \pm}=\chi_{+}-\frac{1}{3}\left(\chi_{+}\right)_{a a},
\end{aligned}
$$

with $\chi=2 B \cdot \operatorname{diag}\left(m_{u}, m_{d}, m_{s}\right), B=|\langle 0|\bar{q} q| 0\rangle| / F^{2}$ and $\left(\chi_{+}\right)_{a a}$ is the trace of $\chi_{+}$.

Following Ref. [19] we neglect the terms $h_{0}, h_{2}$ and $h_{4}$ since they are suppressed in the large- $N_{C}$ limit because of the second flavor trace. The LEC $h_{1}$ can be 

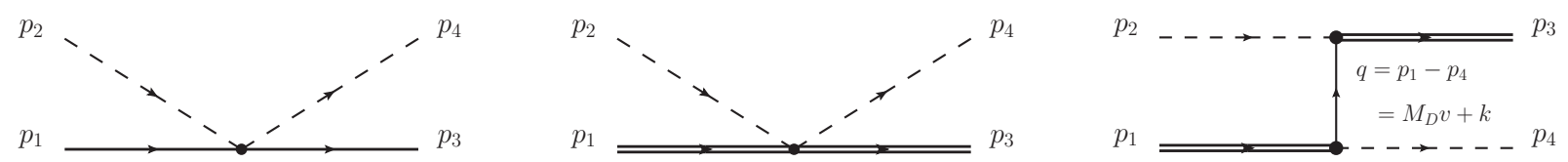

Fig. 1. Diagrams contributing at LO and NLO to Goldstone boson scattering off $D$ - and $D^{\star}$-mesons and the pertinent kinematics. Dashed lines denote Goldstone bosons, solid lines charmed pseudoscalar mesons and solid double lines charmed vector mesons, in order.

determined using the relation $B\left(m_{s}-\hat{m}\right)=\left(M_{K^{0}}^{2}+\right.$ $\left.M_{K^{+}}^{2}\right) / 2-M_{\pi^{0}}^{2}$ as done in [13. We use the value $h_{1}=$ $0.42 \pm 0.00$.

Since we are interested in the masses of the resonances, isospin breaking can be neglected. Hence, we take averaged values for charged and neutral particle masses, and the pion decay constant from the PDG, as listed in the following [29]:

$$
\begin{array}{ll}
M_{D}=1867 \mathrm{MeV}, & M_{D_{s}}=1968 \mathrm{MeV}, \\
M_{D^{*}}=2008 \mathrm{MeV}, & M_{D_{s}^{*}}=2112 \mathrm{MeV}, \\
M_{\pi}=138 \mathrm{MeV}, & M_{K}=496 \mathrm{MeV}, \\
M_{\eta}=548 \mathrm{MeV} . &
\end{array}
$$

We will calculate the scattering of the Goldstone bosons off the pseudoscalar $D$-mesons $\left(0^{+}\right.$channel $)$as well as off vector $D^{*}$-mesons $\left(1^{+}\right.$channel). The diagrams at the order we are working are shown in Fig. 1 The diagrams are evaluated in the isospin basis, the relation between the isospin basis and the particle basis can be found in the Appendix of Ref. [22].

Evaluating the diagrams for the leading order contact interaction we find that the contributions for the $0^{+}$channel and the $1^{+}$channel are the same up to the different masses for the pseudoscalar and vector charmed mesons, as expected from heavy quark spin symmetry:

$$
V_{\mathrm{LO}}^{0^{+}}=V_{\mathrm{LO}}^{1^{+}}=C_{0} \sqrt{M_{1} M_{3}} \frac{1}{2 F^{2}}\left(E_{2}+E_{4}\right) .
$$

The constants $C_{0}$ for the various channels are listed in Tab. 1 Further, $M_{1}$ and $M_{3}$ are the masses of the in-coming and out-going charmed meson, respectively, cf. Fig. 11 and $E_{2}$ and $E_{4}$ are the energies of the incoming and out-going light mesons.

For the NLO contact interactions we again find the same contributions except for the different pseudoscalar and vector charmed meson masses:

$$
\begin{aligned}
V_{\mathrm{NLO}}^{0^{+}}= & V_{\mathrm{NLO}}^{1^{+}} \\
= & \sqrt{M_{1} M_{3}} \frac{1}{2 F^{2}}\left[C_{1} \frac{4}{3} h_{1}\right. \\
& \left.+C_{35}\left(h_{3} p_{2} \cdot p_{4}+2 h_{5} E_{2} E_{4}\right)\right],
\end{aligned}
$$

which also preserves spin symmetry. Its S-wave projection is

$$
\begin{aligned}
V_{s}^{0^{+}}= & V_{s}^{1^{+}} \\
= & \sqrt{M_{1} M_{3}} \frac{2}{F^{2}}\left[C_{0}\left(E_{2}+E_{4}\right)+C_{1} \frac{4}{3} h_{1}\right. \\
& \left.+C_{35} \frac{1}{M_{D}} \tilde{h}_{35} E_{2} E_{4}\right]
\end{aligned}
$$

with $h_{35}=h_{3}+2 h_{5} \equiv \tilde{h}_{35} / M_{D}$. This is the only free parameter at this order. This is fitted to the mass of the $D_{s 0}^{*}(2317)$. We get $\tilde{h}_{35}=0.35$ for the dimensionless parameter, which is of natural size.

The $s$-channel exchange needs a $\mathrm{P}$-wave interaction and thus does not need to be considered. The only contribution from exchange diagrams that does not have an vanishing $\mathrm{S}$-wave projection is the $u-$ channel exchange of a charmed pseudoscalar meson in the $1^{+}$ channel:

$$
\begin{aligned}
V_{\mathrm{PS}-\mathrm{Ex}}^{1^{+}}= & -C_{u} \frac{4 g_{\pi}^{2}}{3 F^{2}}\left[\frac{E_{4}}{M_{1}}|\mathbf{p}|^{2}+\frac{E_{2}}{M_{3}}\left|\mathbf{p}^{\prime}\right|^{2}\right] \\
& \times \frac{1}{(v \cdot k)} \sqrt{M_{1} M_{3}},
\end{aligned}
$$

where the constant $C_{u}$ is also given in Tab. 1 and $\mathbf{p}$ and $\mathbf{p}^{\prime}$ are the three-momenta of the in-coming and out-going mesons in the center-of-mass frame and the energy transfer $(v \cdot k)$ is defined in Fig. 1. Observe, since $k, \mathbf{p}$ and $\mathbf{p}^{\prime}$ are counted of order of the $E_{i}$, this potential appears formally at NLO. Since it acts in the $1^{+}$channel, while there is no counter-part in the $0^{+}$ channel, it thus provides formally the leading source of spin symmetry violation. However, in practice its contribution turns out to be very small, due to three reasons: first of all $g_{\pi}^{2}=0.1$, second, $|\mathbf{p}| \sim \sqrt{M_{K} \epsilon}$, with $\epsilon \equiv M_{D^{*}}+M_{K}-M_{D_{s 1}(2460)} \approx M_{D}+M_{K}-M_{D_{s 0}^{*}(2317)}$ being the binding energy, which is significantly smaller than $M_{K}$ and, most importantly, it does not operate in the $K D^{*} \rightarrow K D^{*}$ channel (in that channel $C_{u}=0$ — c.f. Tab. 11). In total it gives a negligible contribution.

For $\mathrm{SU}(3)$ calculations, the uncertainty from the chiral expansion up to NLO is $\mathscr{O}\left(M_{K}^{2} / \Lambda_{\chi}^{2}\right)$, where $\Lambda_{\chi} \approx$ $4 \pi F_{\pi}$ sets the hard scale in the chiral expansion, compared with the LO contributions. So we use

$$
V^{ \pm}=\left(1 \pm \frac{M_{K}^{2}}{\Lambda_{\chi}^{2}}\right) V_{s}
$$


where $V_{s}$ is the S-wave projection of the full NLO amplitude calculated here for the $0^{+}$and $1^{+}$channel, respectively. A more detailed discussion of the scattering amplitudes is given in the Appendix.

Dynamical generation of bound states from a theory without bound states as fundamental fields is a non-perturbative phenomenon. It cannot be provided by any finite perturbative expansion in the momentum. Thus we have to unitarize, i.e. resum, the amplitude we had obtained so far. Following Ref. 30, the unitarized amplitude can be written as

$$
T(s)=V^{ \pm}(s)\left[1-G(s) \cdot V^{ \pm}(s)\right]^{-1},
$$

where $G(s)$ is the diagonal matrix with non-vanishing elements being loop integrals of the relevant channels - see Eq. (17) below. The bound state mass is found as a pole of the analytically continued unitarized scattering matrix $T$. To be specific, the pole of a bound state, as is the case here, is located on the real axis below threshold on the first Riemann sheet of the complex energy plane (note that because we neglect isospin-breaking, these bound states do not acquire a width). In our approach, both the $D_{s 0}^{*}(2317)$ and the $D_{s 1}(2460)$ appear as poles in the $S=1$ isoscalar $I=0$ channel. Since they couple predominantly to $D K$ and $D^{*} K$, we interpret the $D_{s 0}^{*}(2317)$ and $D_{s 1}(2460)$ as $D K$ and $D^{*} K$ bound states, respectively.

At this point we have to make some comments on the loop matrix. In previous works on this subject [16 17/22, the relativistic two-particle loop function

$$
I=i \int \frac{d^{4} q}{(2 \pi)^{4}} \frac{1}{q^{2}-M_{\phi}^{2}+i \epsilon} \frac{1}{(q-P)^{2}-M_{D}^{2}+i \epsilon}
$$

was used in dimensional regularization with a subtraction constant $a(\mu)$ which absorbs the scale dependence of the integral, where $M_{\phi}$ and $M_{D}$ are the masses of the light meson and charmed meson involved in the loop and $P$ denotes the total momentum. However, this relativistic loop function violates spin symmetry very strongly. Particularly, using the parameters fitted to the mass of the $D_{s 0}^{*}(2317)$, we find the mass of the $D_{s 1}(2460)$ at $2477 \mathrm{MeV}$. From a field theoretical point of view, using a relativistic propagator for a heavy meson and using the Lagrangian from heavy quark expansion simultaneously is inconsistent. There is a well-known problem in relativistic baryon chiral perturbation theory for pion-nucleon scattering, which is closely related to the case here. The relativistic nucleon propagator in a loop explicitly violates power counting, see [31. In our case this effect appears only logarithmically as $\log \left(M_{D}^{2} / \mu^{2}\right)$. However, it induces a violation of spin symmetry in the interaction already at NLO and thus violates the power counting.

There are different ways to deal with this. In [12] a subtraction scheme is used that identifies the scale $\mu$ with the mass of the $D$ - and $D^{*}$-mesons, respectively, and thus the spin symmetry violating terms disappear.
In Refs. [1617] simply different subtraction constants are used for the two channels. In this work we use a static propagator for the heavy meson, as this is consistent with the Lagrangian in the heavy quark expansion. This also allows us to use the same subtraction constant and scale of regularization for both channels. The heavy meson propagator for the vector $D^{*}$-meson then takes the form

$$
\frac{\left\{P^{\mu \nu}, 1\right\}}{q^{2}-M_{D}^{2}+i \epsilon} \rightarrow \frac{\left\{P^{\mu \nu}, 1\right\}}{2 M_{D}(v \cdot k-\Delta+i \epsilon)},
$$

where $P^{\mu \nu}$ is a projector for spin-1. Dropping $\Delta$ in the denominator and using the one in the numerator of Eq. (18) amounts to the propagator for the pseudoscalar $D$-meson.

With this the integral $I$ defined in Eq. (17) turns into

$$
\begin{aligned}
I= & \frac{1}{16 \pi^{2} M_{D}}\left\{\left(P^{0}-M_{D}\right)\left[a(\mu)+\log \left(\frac{M_{\phi}^{2}}{\mu^{2}}\right)\right]\right. \\
& +2 \sqrt{\left(P^{0}-M_{D}\right)^{2}-M_{\phi}^{2}} \cosh ^{-1}\left(\frac{P^{0}-M_{D}}{M_{\phi}}\right) \\
& \left.-2 \pi i\left|\mathbf{p}_{\mathrm{cms}}\right|\right\}+\mathcal{O}\left(\frac{M_{\phi}}{M_{D}}\right),
\end{aligned}
$$

with $a(\mu)$ a subtraction constant, $\mu$ the scale of dimensional regularization, which is fixed to the averaged mass of $D$ and $D^{*}$, and $\left|\mathbf{p}_{\mathrm{cms}}\right|=\sqrt{\left(P_{0}-M_{D}\right)^{2}-M_{\phi}^{2}}$. Note, the use of the modified loop function of Eq. (19) leads only to tiny changes from relativistic effects in the observables discussed in Refs. 2022. The subtraction constant $a(\mu)$ is fitted to the mass of the $D_{s 0}^{*}(2317)$. We find $a(\mu=1936 \mathrm{MeV})=-3.034$. Using the same parameters for the axial-vector channel we find the pole at

$$
M_{D_{s 1}(2460)}=(2459.6 \pm 0.6 \pm 1.8) \mathrm{MeV},
$$

where the first uncertainty stems from the experimental uncertainty for the mass of the $D_{s 0}^{*}(2317)$, which was used for fitting the parameters, and the second $4 \%$ uncertainty estimates the higher orders from the heavy quark expansion - since there is no significant spin symmetry breaking term in the scattering amplitudes at NLO, spin symmetry breaking effect should appear at $\mathscr{O}\left(\left[\Lambda_{\mathrm{QCD}} / m_{c}\right]^{2}\right)$. Hence the second uncertainty can be estimated by $\left(\Lambda_{\mathrm{QCD}} / m_{c}\right)^{2} \epsilon$. In this context it is interesting to note that the spin-symmetry violation induced by $M_{D_{s} *}-M_{D_{s}} \neq M_{D *}-M_{D}$, which is of $\mathrm{N}^{2} \mathrm{LO}$, indeed contributes to $M_{D_{s 1}(2460)}$ less than $1 \mathrm{MeV}$, consistent with the uncertainty estimate.

The result in Eq. (20) is in perfect agreement with the experimental result

$$
M_{D_{s 1}(2460)}^{\exp }=(2459.5 \pm 0.6) \mathrm{MeV} .
$$

To the order we are working instead of $F_{\pi}$ we could as well have used $F_{K}=1.18 F_{\pi}$. However, this change 
Table 1. Coefficients for the amplitudes for all possible channels with total strangeness $S=1$ and total isospin $I=0$. While $C_{0}, C_{1}$, and $C_{35}$ act in both channels $0^{+}$and $1^{+}$, while $C_{u}$ acts solely in $1^{+}$.

\begin{tabular}{|l|c|l|c|c|}
\hline Channel & $C_{0}$ & $C_{1}$ & $C_{35}$ & $C_{u}$ \\
\hline$D K \rightarrow D K$ & -2 & $-4 M_{K}^{2}$ & 2 & 0 \\
$D_{s} \eta \rightarrow D_{s} \eta$ & 0 & $-2\left(2 M_{\eta}^{2}-M_{\pi}^{2}\right)$ & $\frac{4}{3}$ & $\frac{2}{3}$ \\
$D_{s} \eta \rightarrow D K$ & $-\sqrt{3}$ & $-\frac{\sqrt{3}}{2}\left(5 M_{K}^{2}-3 M_{\pi}^{2}\right)$ & $\frac{\sqrt{3}}{3}$ & $-\sqrt{\frac{1}{3}}$ \\
\hline
\end{tabular}

Table 2. Comparison of our predictions of the masses of the $\bar{B} K$ and $\bar{B}^{*} K$ bound states with those in Refs. 12[14[15. All masses are given in MeV. The uncertainties given in the first column originate from the residual scale dependence and an estimate of higher order effects, respectively.

\begin{tabular}{|l|lll|}
\hline & This paper & Ref. [12] & Refs. [14|15] \\
\hline$M_{B_{s 0}^{*}}$ & $5696 \pm 20 \pm 30$ & 5643 & $5725 \pm 39$ \\
$M_{B_{s 1}}$ & $5742 \pm 20 \pm 30$ & 5690 & $5778 \pm 7$ \\
\hline
\end{tabular}

does not have any impact on the mass calculated for the $D_{s 1}(2460)$ once the subtraction constant is readjusted to reproduce the mass for the scalar state.

The formalism used here can easily be applied to the meson sector with open bottom as well. In order to do so, we just have to replace the $D$-mesons with the corresponding $\bar{B}$-mesons. Their masses are 29]:

$$
\begin{aligned}
& M_{\bar{B}}=5279 \mathrm{MeV}, \quad M_{\bar{B}_{s}}=5366 \mathrm{MeV}, \\
& M_{\bar{B}^{*}}=5325 \mathrm{MeV}, \quad M_{\bar{B}_{s}^{*}}=5415 \mathrm{MeV} .
\end{aligned}
$$

Heavy quark flavor symmetry implies that all the other parameters stay the same up to higher order corrections. This allows us to predict the masses of the $\bar{B} K$ and $\bar{B}^{*} K$ bound states in the $(S=1, I=0)$ channel. The predicted masses are given in Table 2, together with a comparison with previous calculations 12 14 15. Our results are in reasonable agreement with the previous ones. In our calculation we have two sources of uncertainties, both shown explicitly in the table. One stems from higher order effects, which may be obtained from multiplying the binding energy by $20 \%$, estimated as $\mathcal{O}\left(\Lambda_{\mathrm{QCD}} / m_{c}\right)$ since heavy flavor symmetry was used to relate the LECs in charm and bottom sectors. The other one originates from the intrinsic scale dependence of the result: To come to a fully renormalization group invariant amplitude, a complete one-loop calculation would have been necessary for the transition amplitude. This, however, is beyond the scope of this paper. Thus, when connecting the charm to the bottom sector, a residual scale dependence remains - to quantify it we varied the scale parameter $\mu$ (see Eq. (19)) from the averaged mass of

\footnotetext{
2 The $B^{(*)}$ mesons in the calculations of Ref. 12 should be understood as $\bar{B}^{(*)}$ mesons which contain a $b$ quark rather than $\bar{b}$.
}

$D$ and $D^{*}$ to that of $\bar{B}$ and $\bar{B}^{*}$, while keeping $a(\mu)$ fixed. Combining the two uncertainties in quadrature gives a total uncertainty $40 \mathrm{MeV}$ which is about 1 $\%$ for the masses. When again switching from $F_{\pi}$ to $F_{K}$ as the strength parameter of the interaction (c.f. Eq. (4) and sentences below), the predicted masses change by $8 \mathrm{MeV}$ only, well consistent with our uncertainty estimates.

It should be stressed that the uncertainties quoted for $M_{B_{s 0}^{*}}$ and $M_{B_{s 1}}$ are highly correlated. As already explained for the charmed system, within the molecular scenario the relation

$$
M_{B_{s 1}}-M_{B_{s 0}^{*}} \simeq M_{B^{*}}-M_{B}
$$

should hold up to corrections of $\mathscr{O}\left(\left[\Lambda_{Q C D} / m_{b}\right]^{2}\right)-$ c.f. discussion below Eqs. (14) and (20). Thus we predict

$$
M_{B_{s 1}}-M_{B_{s 0}^{*}}=46 \pm 0.4 \pm 1 \mathrm{MeV},
$$

where the first uncertainty comes from the current experimental uncertainty in $M_{B^{*}}-M_{B}$ and the second from the estimated spin breaking effects in the formation of the molecule. Clearly, all mass differences deduced from Tab. 2 are consistent with this value.

$M_{B_{s 1}}$ and $M_{B_{s 0}^{*}}$ have not been measured so far. Note that their existence in the deduced mass range and, especially, with the mass splitting of Eq. (23), is a crucial and highly non-trivial test for the theory 2 presented and especially for the molecular nature of both states.

\section{Chiral extrapolations}

To test the nature of the resonances besides further experimental data we can also compare our results to lattice calculations (for a corresponding study in the light meson sector see Ref. 32]). To do so, we extend the calculations from the physical world to unphysical quark masses which are frequently used in lattice calculations. Varying the light quark masses is equivalent to varying the pion mass. Although the physical strange quark mass is nowadays routinely used in lattice calculations, we emphasize that by varying the strange quark mass, or equivalently varying the kaon mass, one can learn a lot about the nature of some hadrons, as will be discussed below. So we have to express all results in terms of pion and kaon masses, respectively. In the following chapter only the charmed 
mesons are discussed explicitly, however, analogous arguments apply to their bottom partners.

In order to proceed we need to assume that the subtraction constant $a(\mu)$ does not depend on the light quark masses. We stress, however, that even allowing for a quark mass dependence of $a(\mu)$ would not change the general features of the results, but might slightly enhance the uncertainties.

\subsection{Pion mass dependence}

Lattice QCD calculations are often performed at larger quark masses than realized in nature - for the $K D$ system of interest here exploratory lattice studies are presented in Ref. 33. In addition, as we will argue here, the quark mass dependence of a state contains important information on its nature. Varying $u$ and $d$ quark masses can be expressed by varying the pion mass. In 34, the charmed meson masses were expanded up to one loop order in the chiral expansion. Nevertheless for our purposes the expansion up to $\mathscr{O}\left(M_{\pi}^{2}\right)$ is sufficient.

Using the Lagrangian given in Eq. (8) we find the NLO correction to the charmed meson masses to be

$$
\delta M_{D^{(*)}}^{2}=4 h_{1} B \hat{m}, \quad \delta M_{D_{s}^{(*)}}^{2}=4 h_{1} B m_{s},
$$

with $\hat{m}=\left(m_{u}+m_{d}\right) / 2$ the average light quark mass. When studying the pion mass dependence, we consider the physical value for the strange quark mass here, thus we can use the physical mass for the $D_{s}^{(*)}$. Using $M_{\pi}^{2}=2 B \hat{m}$ yields, up to $\mathscr{O}\left(M_{\pi}^{2}\right)$,

$$
M_{D^{(*)}}=\stackrel{\circ}{M}_{D^{(*)}}+h_{1} \frac{M_{\pi}^{2}}{\stackrel{\circ}{M}_{D^{(*)}}},
$$

where $\stackrel{\circ}{M}_{D^{(*)}}$ is the charmed meson mass in the $\mathrm{SU}(2)$ chiral limit $m_{u}=m_{d}=0$ with $m_{s}$ fixed at its physical value. For the kaon and the eta mass we can find similar expressions by using $M_{K}^{2}=B\left(\hat{m}+m_{s}\right)$, $\stackrel{\circ}{M}_{K}^{2}=B m_{s}$ and $M_{\eta}^{2}=B \frac{2}{3}\left(\hat{m}+2 m_{s}\right), \stackrel{\circ}{M}_{\eta}^{2}=B \frac{4}{3} m_{s}$ from the LO chiral expansion:

$$
M_{K}=\stackrel{\circ}{M}_{K}+\frac{M_{\pi}^{2}}{4 \stackrel{\circ}{M}_{K}}, \quad M_{\eta}=\stackrel{\circ}{M}_{\eta}+\frac{M_{\pi}^{2}}{6 \stackrel{\circ}{M}_{\eta}} .
$$

In Fig. 2 we show the mass of both resonances, $D_{s 0}^{*}(2317)$ and $D_{s 1}(2460)$, as well as their binding energies as a function of the pion mass. Note that the observed rather strong pion mass dependence is specific for a molecular state. The corresponding dependence for a quark state should be a lot weaker. To understand this, one notices that a pure $c \bar{s}$ state does not contain any $u, \bar{u}, d, \bar{d}$ quarks. The leading term containing these light quarks is $1 / N_{c}$ suppressed. Thus, for the quark state, the pion mass dependence can only enter via $D^{(*)} K$ loops - as in case of the molecular state. However, their contribution should be a lot smaller for the quark state than for the molecular state. To see this, observe that the loop contributions from a particular meson pair is proportional to the squared coupling of that meson pair to the resonance. As shown in Refs. 23/24, this coupling is proportional to the probability to find the molecular state in the physical state. Thus, the pion mass dependence for $D_{s 0}^{*}(2317)$ and $D_{s 1}(2460)$ should be maximal if both are pure molecules. This case is depicted in Fig. 2. On the other hand it should be very weak for the admittedly unrealistic scenario of a pure quark state. Note that the mentioned relation between the coupling and the structure of the state holds only in leading order in an expansion in $\sqrt{M_{K} \epsilon} / \beta$ (see Ref. [35] for a detailed discussion), with the inverse range of forces $\beta \sim m_{\rho}$. For the scalar and axial vector open charm mesons this gives an uncertainty of the order of $20 \%$. As a result of the larger binding, for the bottom analogs we even find $30 \%$. In addition in the analysis we neglected terms of $\mathcal{O}\left(1 / N_{c}\right)$. Thus, from this kind of analysis at most statements like 'the state is predominantly molecular/compact' are possible.

Furthermore we notice that the plots show an almost identical behavior in the scalar and the axialvector channel. So the spin symmetry breaking effects are only very weak here. We see that the binding energy in both cases varies from about $40 \mathrm{MeV}$ to about $80 \mathrm{MeV}$.

\subsection{Kaon mass dependence}

Before going into details of calculations, let us make some general statements about the $M_{K}$-dependence of the mass of a bound state of a kaon and some other hadron. The mass of such a kaonic bound state is given by

$$
M=M_{K}+M_{h}-\epsilon,
$$

where $M_{h}$ is the mass of the other hadron, and $\epsilon$ denotes the binding energy. Although both $M_{h}$ and $\epsilon$ have some kaon mass dependence, it is expected to be a lot weaker than that of the kaon itself. Thus, the important implication of this simple formula is that the leading kaon mass dependence of a kaonhadron bound state is linear, and the slope is unity. The only exception to this argument is if the other hadron is also a kaon or anti-kaon. 3 In this case, the leading kaon mass dependence is still linear but with the slope being changed to two. Hence, as for the $D K$ and $D^{*} K$ bound states discussed here, we expect that their masses are linear in the kaon mass, and the slope is approximately one. As we will see, our explicit calculations confirm this expectation.

\footnotetext{
3 The $f_{0}(980)$ was proposed to be such a $K \bar{K}$ bound state $36[24$.
} 

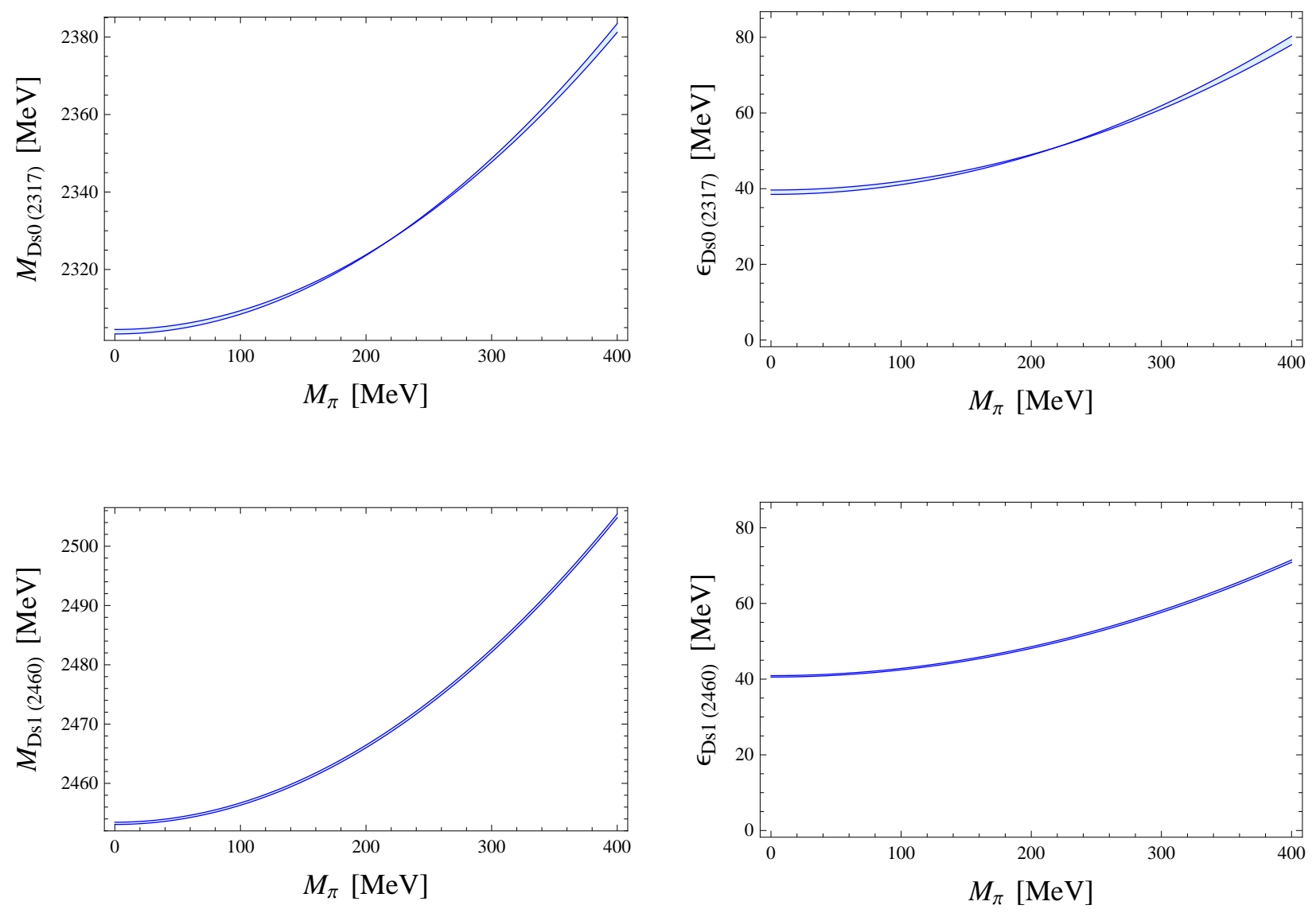

Fig. 2. The masses and binding energies of the $D_{s 0}^{*}(2317)$ and the $D_{s 1}(2460)$ as a function of the pion mass.

For calculating the kaon mass dependence, we use physical masses for the pion and the charmed mesons without strangeness. 4 To express the strange quark mass dependence in terms of kaon masses, we write

$$
M_{K}^{2}=B\left(m_{s}+\hat{m}\right), \quad \stackrel{\circ}{M}_{K}^{2}=B \hat{m}=\frac{1}{2} M_{\pi}^{2},
$$

where $\stackrel{\circ}{M}_{K}^{2}$ is the mass of the kaon in the limit $m_{s}=0$. The charmed strange meson mass is

$$
M_{D_{s}^{(*)}}=\stackrel{\circ}{M}_{D_{s}^{(*)}}+2 h_{1} \frac{M_{K}^{2}}{\stackrel{\circ}{M}_{D_{s}^{(*)}}}-h_{1} \frac{M_{\pi}^{2}}{\stackrel{\circ}{M}_{D_{s}^{(*)}}},
$$

where $\stackrel{\circ}{M}_{D_{s}^{(*)}}$ is the charmed strange meson mass in the limit $m_{s}=0$. Finally the eta mass is given to this order by the Gell-Mann-Okubo relation

$$
M_{\eta}^{2}=B\left(\frac{4}{3} m_{s}+\hat{m}\right)=\frac{4}{3} M_{K}^{2}-\frac{1}{3} M_{\pi}^{2} .
$$

\footnotetext{
${ }^{4}$ Certainly, lattice simulations use unphysical values for up and down quarks. However, the conclusion of this subsection will not be affected by the value of pion mass. For definiteness, we choose to use physical masses for the pion and non-strange charmed mesons.
}

For analyzing the kaon mass dependence we calculate the masses of the $D_{s 0}^{*}(2317)$ and $D_{s 1}(2460)$ and their binding energies as before. The corresponding results are shown in Fig. 3. Furthermore we also show the $D^{(*)} K$ threshold in dependence on the kaon mass.

For the $D_{s 0}^{*}(2317)$, we find that the kaon mass dependence of the mass is almost perfectly linear, especially in the region $M_{K}=300-500 \mathrm{MeV}$. For higher strange quark masses the chiral expansion is no longer valid and the results become less reliable. The kaon mass dependence of the mass and the $D K$ threshold have a similar slope. The slope of the threshold is one, since the $D$-meson mass is independent of $M_{K}$ to the order we are working, and the slope of $M_{D_{s 0}^{*}(2317)}$ is about 0.85 . This finding is perfectly consistent with the expectation formulated in the beginning of this subsection. The deviation of the slope from one can be understood from the kaon mass dependence of the binding energy (see right column of Fig. 3) and from the effects of the coupling to the $D_{s} \eta$ channel. The situation for the $D_{s 1}(2460)$ is similar.

On the contrary, if $D_{s 0}^{*}(2317)$ and $D_{s 1}(2460)$ are assumed to be quark states, their masses necessarily depend quadratically on the kaon mass - analogous to Eq. (29). Thus, an extraction of the kaon mass dependence of the masses of $D_{s 0}^{*}(2317)$ and $D_{s 1}(2460)$ 

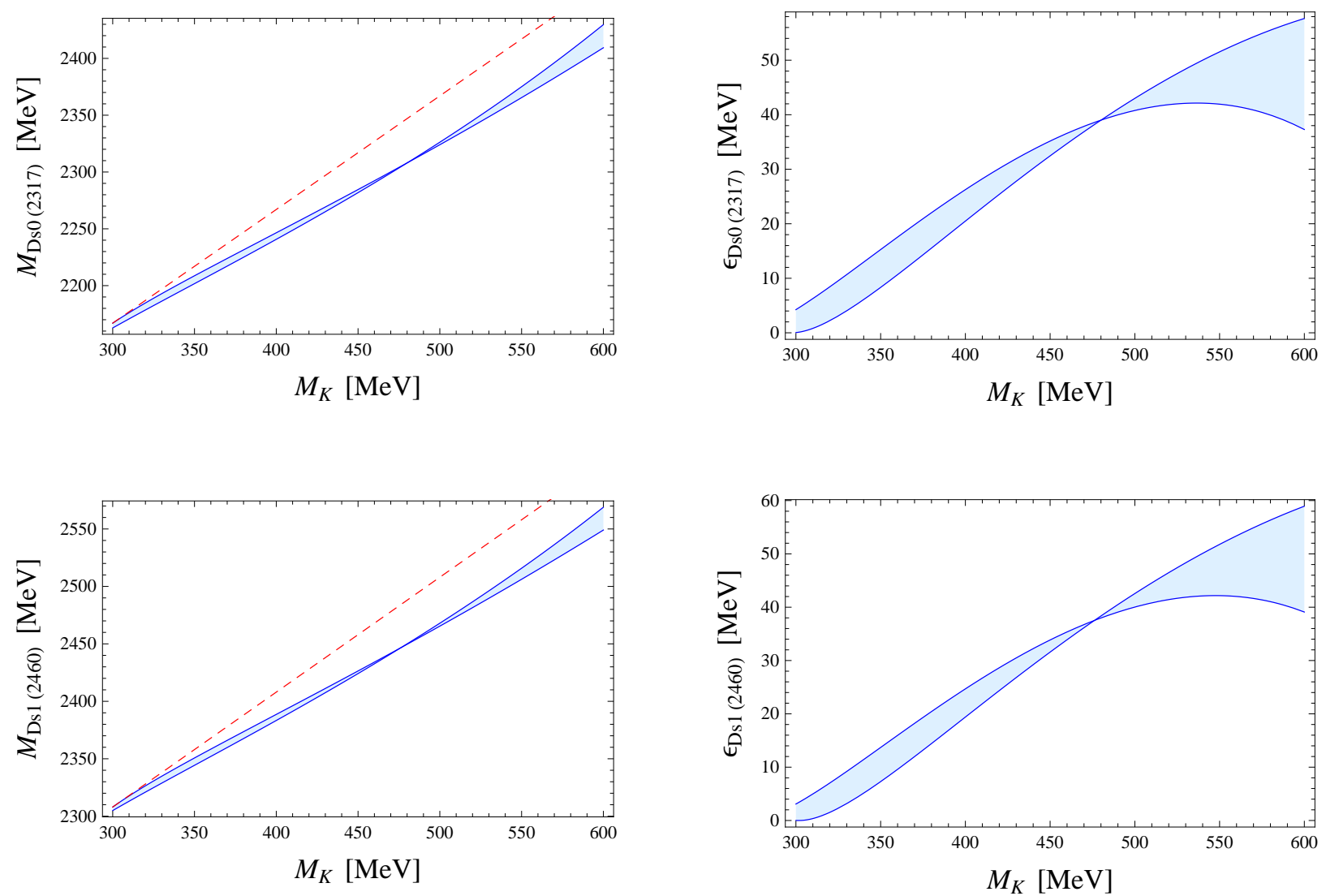

Fig. 3. The blue band shows the masses and binding energies of $D_{s 0}^{*}(2317)$ and $D_{s 1}(2460)$ in dependence of the kaon mass, the red dashed line shows the threshold.

from lattice data is of high interest to pin down the nature of these states.

\section{Summary}

In this paper we used unitarized heavy meson chiral perturbation theory to calculate the masses of the resonances $D_{s 0}^{*}(2317)$ and $D_{s 1}(2460)$ as well as their bottom analogs $B_{s 0}^{*}$ and $B_{s 1}$. The results for the latter turned out to be consistent with previous analyses. Especially we predict $M_{B_{s 1}}-M_{B_{s 0}^{*}}=46 \pm 1 \mathrm{MeV}$, where we added the uncertainties of Eq. (23) in quadrature. In addition, calculations were performed with varying pion and kaon masses. Within the molecular picture used here, we found a linear dependence of the resonance masses on the kaon mass with the slope being approximately unity and a strong quadratic dependence on the pion mass. We argued that, if both states are assumed to be elementary quark states, the mentioned dependences would be qualitatively different: while the pion mass dependence should still be quadratic, it should be a lot weaker, the kaon mass dependence should come out quadratic as well. Thus, especially an investigation of the kaon mass dependence of the $D_{s 0}^{*}(2317)$ and $D_{s 1}(2460)$ or their bottom analogs within lattice QCD would be very interesting in order to determine the nature of these resonances.

\section{Acknowledgments}

This work is partially supported by the Helmholtz Association through funds provided to the Virtual Institute "Spin and strong QCD" (VH-VI-231) and by the DFG (TR 16, "Subnuclear Structure of Matter"), the European Community-Research Infrastructure Integrating Activity "Study of Strongly Interacting Matter" (acronym HadronPhysics2, Grant Agreement n. 227431) under the FP7 of the EU, and the BMBF (grant 06BN9006).

\section{A Amplitudes}

Here we give all the contributing amplitudes and their S-wave projections. In all the following expressions, $p_{1(3)}$ and $p_{2(4)}$ denote the momentum of the in-coming (out-going) heavy meson and light meson, respectively, the heavy meson mass is given by $M_{1(3)}$, and the energy of the light meson is given by $E_{2(4)}$. The leading order contact interactions are purely S-wave by 
construction and identical up to the masses for both channels:

$$
\begin{gathered}
T_{\mathrm{LO}}^{0^{+}}=\sqrt{M_{1} M_{3}} C_{0} \frac{1}{2 F^{2}}\left(E_{2}+E_{4}\right) \\
T_{\mathrm{LO}}^{1^{+}}=-\sqrt{M_{1} M_{3}}\left(\epsilon \cdot \epsilon^{*}\right) C_{0} \frac{1}{2 F^{2}}\left(E_{2}+E_{4}\right) \\
=\sqrt{M_{1} M_{3}} C_{0} \frac{1}{2 F^{2}}\left(E_{2}+E_{4}\right),
\end{gathered}
$$

where $\epsilon$ is the polarization vector of the $D^{*}$.

The NLO contact interaction reads:

$$
\begin{aligned}
T_{\mathrm{NLO}}^{0^{+}}= & \sqrt{M_{1} M_{3}}\left[C_{1} \frac{2}{3 F^{2}} h_{1}\right. \\
& \left.+C_{35} \frac{2}{F^{2}}\left(h_{3} p_{2} \cdot p_{4}+2 h_{5} E_{2} E_{4}\right)\right] \\
T_{\mathrm{NLO}}^{1^{+}}= & -\sqrt{M_{1} M_{3}} \epsilon \cdot \epsilon^{*}\left[C_{1} \frac{2}{3 F^{2}} h_{1}\right. \\
& \left.+C_{35} \frac{2}{F^{2}}\left(h_{3} p_{2} \cdot p_{4}+2 h_{5} E_{2} E_{4}\right)\right] \\
= & \sqrt{M_{1} M_{3}}\left[C_{1} \frac{2}{3 F^{2}} h_{1}\right. \\
& \left.+C_{35} \frac{2}{F^{2}}\left(h_{3} p_{2} \cdot p_{4}+2 h_{5} E_{2} E_{4}\right)\right] .
\end{aligned}
$$

The matrix element for the exchange of charmed vector mesons in the $1^{+}$channel is zero at this order:

$$
\begin{aligned}
T_{\text {Vector-Ex }}^{1^{+}} & =\frac{4 g_{\pi}^{2}}{F^{2}} \frac{1}{2 v \cdot k}\left(\epsilon^{*} \cdot \mathbf{p} \epsilon \cdot \mathbf{p}^{\prime}-\epsilon^{*} \cdot \epsilon \mathbf{p} \cdot \mathbf{p}^{\prime}\right) \\
& =0
\end{aligned}
$$

where $\epsilon$ is the spatial component of the polarization vector of the $D^{*}$, and $\mathbf{p}^{(\prime)}$ denotes the three-momentum of the in-coming (out-going) heavy meson in the centerof-mass frame and $k$ is the residual momentum of the exchanged heavy meson (c.f. Fig. 11).

The exchange of charmed vector mesons in the $0^{+}$ channel gives a non-vanishing contribution:

$$
T_{\text {Vector-Ex }}^{0^{+}}=-8 C_{u} \sqrt{M_{1} M_{3}} \frac{g_{\pi}^{2}}{F^{2}} \frac{\mathbf{p} \cdot \mathbf{p}^{\prime}}{2 v \cdot k}
$$

But since it is purely $\mathrm{P}$-wave, the $\mathrm{S}$-wave projection vanishes. Finally we consider the exchange of charmed pseudoscalar mesons in the $1^{+}$channel:

$$
\begin{aligned}
T_{\mathrm{PS}-\mathrm{Ex}}^{1^{+}}= & -C_{u} \frac{8 g_{\pi}^{2}}{F^{2}}\left(\epsilon^{*} \cdot p_{2}\right)\left(\epsilon \cdot p_{4}\right) \frac{1}{2 v \cdot k} \sqrt{M_{1} M_{3}} \\
= & -C_{u} \frac{8 g_{\pi}^{2}}{F^{2}}\left[\frac{E_{4}}{M_{1}}|\mathbf{p}|^{2} \cos ^{2} \theta+\frac{E_{2}}{M_{3}}\left|\mathbf{p}^{\prime}\right|^{2} \cos ^{2} \theta^{\prime}\right. \\
& \left.+\left(\frac{E_{2} E_{4}}{M_{1} M_{3}}|\mathbf{p}|\left|\mathbf{p}^{\prime}\right|+|\mathbf{p}|\left|\mathbf{p}^{\prime}\right|\right) \cos \theta \cos \theta^{\prime}\right] \\
& \times \frac{1}{2(v \cdot k)} \sqrt{M_{1} M_{3}},
\end{aligned}
$$

where $\theta\left(\theta^{\prime}\right)$ is the angle between $\mathbf{p}\left(\mathbf{p}^{\prime}\right)$ and the quantization axis used for the polarization vectors. The projections $\lambda$ and $\lambda^{\prime}$ of the polarization vectors is conserved for $S$-wave scattering. The expression given holds for $\lambda=\lambda^{\prime}=0$. This gives the only non-vanishing S-wave projection from the exchange diagrams:

$$
\begin{aligned}
T_{\mathrm{PS}-\mathrm{Ex}}^{1^{+}}= & -C_{u} \frac{4 g_{\pi}^{2}}{3 F^{2}}\left(\frac{E_{4}}{M_{1}}|\mathbf{p}|^{2}+\frac{E_{2}}{M_{3}}\left|\mathbf{p}^{\prime}\right|^{2}\right) \\
& \times \frac{1}{(v \cdot k)} \sqrt{M_{1} M_{3}} .
\end{aligned}
$$

As explained in the main text, this contribution is formally of NLO, however, for the resonances discussed it gives a negligible contribution.

\section{References}

1. B. Aubert et al. [BABAR Collaboration], Phys. Rev. Lett. 90, 242001 (2003) (arXiv:hep-ex/0304021).

2. D. Besson et al. [CLEO Collaboration], Phys. Rev. D 68, 032002 (2003) [Erratum-ibid. D 75, 119908 (2007)] (arXiv:hep-ex/0305100).

3. S. Godfrey and N. Isgur, Phys. Rev. D 32, 189 (1985).

4. W. A. Bardeen, E. J. Eichten and C. T. Hill, Phys. Rev. D 68, 054024 (2003) (arXiv:hep-ph/0305049).

5. M. A. Nowak, M. Rho and I. Zahed, Acta Phys. Polon. B 35, 2377 (2004) (arXiv:hep-ph/0307102).

6. T. Mehen and R. P. Springer, Phys. Rev. D 72, 034006 (2005) (arXiv:hep-ph/0503134).

7. F.-K. Guo, C. Hanhart and U.-G. Meißner, Phys. Rev. Lett. 102, 242004 (2009) (arXiv:0904.3338 [hep-ph]).

8. T. Barnes, F. E. Close and H. J. Lipkin, Phys. Rev. D 68, 054006 (2003) (arXiv:hep-ph/0305025).

9. E. van Beveren and G. Rupp, Phys. Rev. Lett. 91, 012003 (2003) (arXiv:hep-ph/0305035).

10. E. S. Swanson, Phys. Rept. 429, 243 (2006) (arXiv:hep-ph/0601110).

11. S. L. Zhu, Int. J. Mod. Phys. E 17, 283 (2008) (arXiv:hep-ph/0703225).

12. E. E. Kolomeitsev and M. F. M. Lutz, Phys. Lett. B 582, 39 (2004) (arXiv:hep-ph/0307133).

13. J. Hofmann and M. F. M. Lutz, Nucl. Phys. A 733, 142 (2004) arXiv:hep-ph/0308263).

14. F.-K. Guo, P. N. Shen, H. C. Chiang, R. G. Ping and B. S. Zou, Phys. Lett. B 641, 278 (2006) (arXiv:hep-ph/0603072).

15. F.-K. Guo, P. N. Shen and H. C. Chiang, Phys. Lett. B 647, 133 (2007) (arXiv:hep-ph/0610008).

16. D. Gamermann, E. Oset, D. Strottman and M. J. Vicente Vacas, Phys. Rev. D 76, 074016 (2007) (arXiv:hep-ph/0612179).

17. D. Gamermann and E. Oset, Eur. Phys. J. A 33, 119 (2007) (arXiv:0704.2314 [hep-ph]).

18. A. Faessler, T. Gutsche, V. E. Lyubovitskij and Y. L. Ma, Phys. Rev. D 76, 014005 (2007) (arXiv:0705.0254 [hep-ph]).

19. M. F. M. Lutz and M. Soyeur, Nucl. Phys. A 813, 14 (2008) (arXiv:0710.1545 [hep-ph]). 
20. F.-K. Guo, C. Hanhart, S. Krewald and U.G. Meißner, Phys. Lett. B 666, 251 (2008) (arXiv:0806.3374 [hep-ph]).

21. P. Colangelo and F. De Fazio, Phys. Lett. B 570, 180 (2003) (arXiv:hep-ph/0305140).

22. F.-K. Guo, C. Hanhart and U.-G. Meißner, Eur. Phys. J. A 40, 171 (2009) (arXiv:0901.1597 [hep-ph]).

23. S. Weinberg, Phys. Rev. 137, B672 (1965).

24. V. Baru, J. Haidenbauer, C. Hanhart, Yu. Kalashnikova and A. E. Kudryavtsev, Phys. Lett. B 586, 53 (2004) (arXiv:hep-ph/0308129).

25. G. Burdman and J. F. Donoghue, Phys. Lett. B 280, $287(1992)$

26. M. B. Wise, Phys. Rev. D 45, 2188 (1992).

27. T. M. Yan, H. Y. Cheng, C. Y. Cheung, G. L. Lin, Y. C. Lin and H. L. Yu, Phys. Rev. D 46, 1148 (1992); 55, $5851(1997)(\mathrm{E})$.

28. A. F. Falk, H. Georgi, B. Grinstein and M. B. Wise, Nucl. Phys. B 343, 1 (1990).

29. K. Nakamura et al. [Particle Data Group], J. Phys. G 37, 075021 (2010).

30. J. A. Oller and U.-G. Meißner, Phys. Lett. B 500, 263 (2001) (arXiv:hep-ph/0011146).

31. J. Gasser, M. E. Sainio and A. Svarc, Nucl. Phys. B 307, 779 (1988).

32. C. Hanhart, J. R. Pelaez and G. Rios, Phys. Rev. Lett. 100 (2008) 152001 arXiv:0801.2871 [hep-ph]].

33. L. Liu, H. W. Lin and K. Orginos, PoS LATTICE2008, 112 (2008) (arXiv:0810.5412 [hep-lat]).

34. E. E. Jenkins, Nucl. Phys. B 412, 181 (1994) (arXiv:hep-ph/9212295).

35. C. Hanhart, Yu. S. Kalashnikova, A. E. Kudryavtsev and A. V. Nefediev, Phys. Rev. D 75 (2007) 074015 arXiv:hep-ph/0701214.

36. J. D. Weinstein and N. Isgur, Phys. Rev. D 41, 2236 (1990). 